\title{
Body Composition Analysis of Computed Tomography Scans in Clinical Populations: The Role of Deep Learning
}

\author{
Michael T. Paris \\ Department of Kinesiology, University of Waterloo, Waterloo, ON, Canada
}

\section{Keywords}

Automated body composition analysis - Computed tomography . Deep learning $\cdot$ Sarcopenia

\begin{abstract}
Background: Body composition is increasingly being recognized as an important prognostic factor for health outcomes across cancer, liver cirrhosis, and critically ill patients. Computed tomography (CT) scans, when taken as part of routine care, provide an excellent opportunity to precisely measure the quantity and quality of skeletal muscle and adipose tissue. However, manual analysis of CT scans is costly and timeintensive, limiting the widespread adoption of CT-based measurements of body composition. Summary: Advances in deep learning have demonstrated excellent success in biomedical image analysis. Several recent publications have demonstrated excellent accuracy in comparison to human raters for the measurement of skeletal muscle, visceral adipose, and subcutaneous adipose tissue from the lumbar vertebrae region, indicating that analysis of body composition may be successfully automated using deep neural networks. Key Messages: The high accuracy and drastically improved speed of CT body composition analysis ( $<1 \mathrm{~s} / \mathrm{scan}$ for neural networks vs. $15 \mathrm{~min} / \mathrm{scan}$ for human analysis) suggest that neural networks may aid researchers and clinicians in better understanding the role of body composition in clinical pop-
\end{abstract}

ulations by enabling cost-effective, large-scale research studies. As the role of body composition in clinical settings and the field of automated analysis advance, it will be critical to examine how clinicians interact with these systems and to evaluate whether these technologies are beneficial in improving treatment and health outcomes for patients.

(c) 2019 The Author(s)

Published by S. Karger AG, Basel

\section{Body Composition Assessment in Clinical Populations}

Body composition, defined as the proportions of skeletal muscle and adipose tissue, is increasingly being recognized by researchers and clinicians as a critical component in disease prognosis and treatment planning

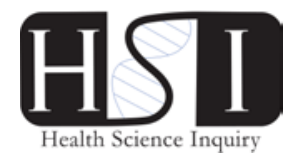

This article was selected as the top submission to our partner journal, Health Science Inquiry (www.healthscienceinquiry.com), a student-led publication platform that seeks to publish the views of Canadian graduate students on pertinent issues in the health sciences.

\begin{tabular}{ll}
\hline KARGER & $\begin{array}{l}\text { C 2019 The Author(s) } \\
\text { Published by S. Karger AG, Basel Oper }\end{array}$ \\
E-Mail karger@karger.com & This article is licensed under the Creative Commons Attribution- \\
wwww.karger.com/lfg & $\begin{array}{l}\text { NonCommercial-NoDerivatives 4.0 International License (CC BY- } \\
\text { NC-ND) (http://www.karger.com/Services/OpenAccessLicense). } \\
\text { Usage and distribution for commercial purposes as well as any dis- } \\
\text { tribution of modified material requires written permission. }\end{array}$
\end{tabular}


Fig. 1. Computed tomography body composition analysis of the third lumbar vertebrae.

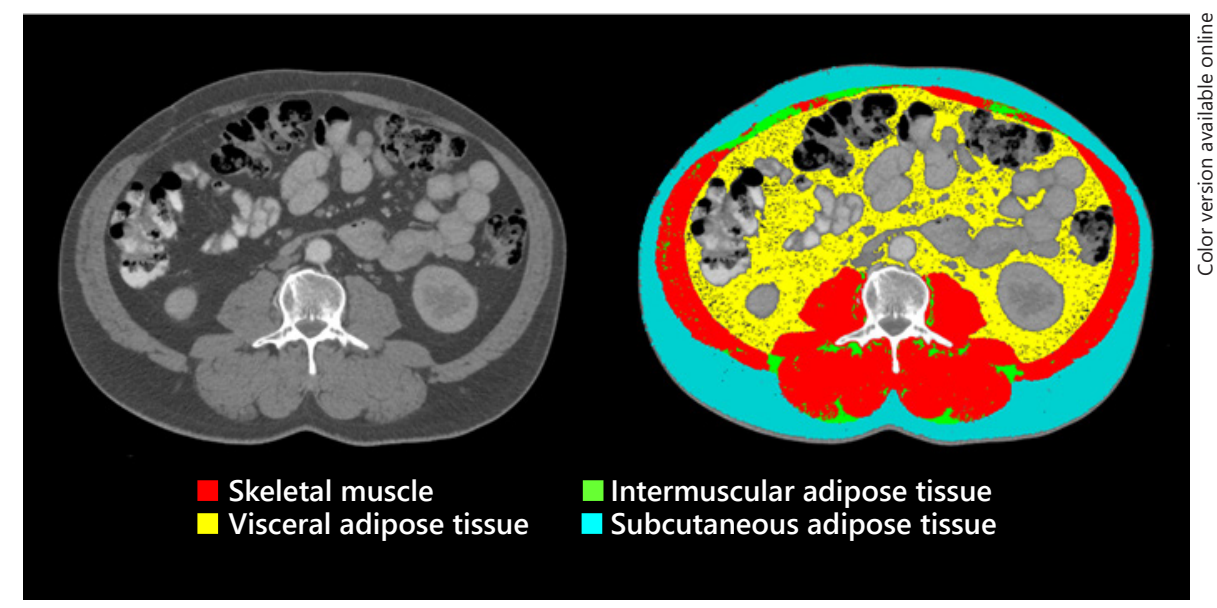

[1]. Several clinical populations, including liver cirrhosis, various cancer, and critically ill patients, display worse clinical outcomes in the presence of abnormal body composition features (e.g., low skeletal muscle mass) $[2,3]$. Identifying patients with these abnormal body composition features can be a challenge in clinical settings due to a lack of access to precise body composition modalities (e.g., dual-energy X-ray absorptiometry) or technical expertise, limiting assessments to typically using body mass index. However, body mass index is a crude measure of body size which cannot distinguish skeletal muscle or adipose tissue from body weight. In contrast, computed tomography (CT) scans, which are often acquired for clinical purposes (e.g., monitoring disease progression), can be retrospectively analyzed to precisely segment skeletal muscle, intermuscular adipose tissue, visceral adipose tissue, and subcutaneous adipose tissue (Fig. 1) [4]. Typically, a single two-dimensional CT scan from the lumbar vertebrae (e.g., third lumbar vertebrae) is used as a consistent bony landmark for body composition analysis, as the skeletal muscles and adipose tissue in this region display strong associations with whole-body metrics of body composition [5]. While retrospective analysis of these scans has been instrumental in our understanding of the intricate relationships between body composition and clinical outcomes, analysis of these scans requires laborious manual segmentation of specific tissues using specialized software. Segmentation of a single scan may require 1520 min of analysis time, which can rapidly become timeand cost-prohibitive in large-scale investigations (e.g., $4,000 \mathrm{CT}$ scans require approximately $1,000-1,300 \mathrm{~h}$ of analysis time).

Deep Learning and Body Composition in Clinical Populations

\section{Overview of Automated Frameworks for Body Composition Analysis of CT Scans}

Development of a framework for the automated analysis of CT scans for body composition would enable clinicians and researchers to better leverage the immense repository of available scans (approximately 5.6 million CT scans performed per year in Canada [6]) for investigating the role of body composition in clinical populations. While a few approaches have proposed sophisticated, hand-crafted features for automatically analyzing CT scans [7], recent advances in deep learning have demonstrated immense success in biomedical image segmentation. Deep learning is a subfield of machine learning which utilizes deep neural networks to make predictions (e.g., segmentation maps) based on input data (e.g., CT scans). A basic neural network is a series of stacked layers containing multiple neurons, which process (using simple mathematical operations) and then transmit information to subsequent connected layers [8]. A deep neural network is composed of an input layer, multiple hidden layers, and an output layer [9]. The hidden layers are fully connected, meaning that the outputs of these neurons are shared with all neurons of the subsequent layers (i.e., the neuron outputs of one layer become the input to neurons in the next layer), amplifying the important features of the input data for classification in the output layer. The final output layer assigns a probability for a label for classification.

Advances in learning algorithms and network architectures have led to variants of basic neural networks that are optimized for specific tasks. Convolutional neural networks (CNNs) are a class of deep neural networks most commonly applied to computer vision problems

Lifestyle Genomics 2020;13:28-31 
Table 1. Deep learning approaches for body composition analysis of the third lumbar vertebrae of computed tomography scans

\begin{tabular}{|c|c|c|c|c|c|c|}
\hline \multirow[t]{2}{*}{ Reference } & \multirow{2}{*}{$\begin{array}{l}\text { Time per } \\
\text { scan, s }\end{array}$} & \multirow[t]{2}{*}{ Training cohort $(n)$} & \multirow[t]{2}{*}{ Testing cohort $(n)$} & \multicolumn{3}{|c|}{ Testing accuracy } \\
\hline & & & & $\begin{array}{l}\text { muscle } \\
\text { Dice }\end{array}$ & $\begin{array}{l}\text { visceral } \\
\text { adipose Dice }\end{array}$ & $\begin{array}{l}\text { subcutaneous } \\
\text { adipose Dice }\end{array}$ \\
\hline Lee et al. [13], 2017 & 0.17 & lung cancer (250) & lung cancer (150) & 0.93 & - & - \\
\hline Wang et al. [14], 2017 & - & ovarian cancer $(20)$ & ovarian cancer $(20)$ & - & 0.915 & 0.980 \\
\hline Bridge et al. [15], 2018 & 0.22 & pancreatic cancer (506) & pancreatic cancer (89) & 0.968 & 0.954 & 0.984 \\
\hline Weston et al. [16], 2019 & - & various cancers $(2,430)$ & various cancers $(2,639)$ & $0.92-0.96$ & 0.94 & $0.94-0.98$ \\
\hline Dabiri et al. [17], 2019 & 0.20 & various cancers $(6,221)$ & various cancers $(2,958)$ & 0.981 & - & - \\
\hline Burns et al. [18], 2019 & - & older adults (51) & older adults (51) & 0.930 & - & - \\
\hline
\end{tabular}

(e.g., image classification, image segmentation). CNNs implement convolutional layers in place of fully connected hidden layers, which assemble simple image features into complex patterns in deeper layers [9]. LeCun et al. [10] pioneered a seven-layer CNN in 1998 to classify handwritten digits, demonstrating the potential for deep learning for image classification tasks. In 2015, Long et al. [11] and Ronneberger et al. [12] introduced the fully convolutional network (FCN) and UNet architectures, which are variants of CNNs focused on the task of image segmentation rather than classification.

Several recent publications have applied FCN and UNet architectures to automate segmentation of skeletal muscle and adipose tissue from CT scans (Table 1) [1318]. These deep learning approaches leverage large datasets of CT scans that have been previously analyzed by human raters and utilize neural networks to learn image features that can best segment muscle and adipose tissue. These networks can then be used to predict the segmentation maps for skeletal muscle and adipose tissue of new CT scans. To assess the performance of these automated approaches, the agreement between human-analyzed and predicted segmentations is often quantified using the Dice similarity coefficient, a statistic which measures the degree of overlap between two segmentations maps ( 0 indicating no overlap, 1 indicating perfect overlap). Network performance should be assessed on data that were not used during the training process, as neural networks tend to overfit training data. Assessment of network performance is often achieved using several different approaches, including cross-validation, splitting the original data into training and test sets, and testing the accuracy on an independent dataset.

\section{Deep Learning and CT-Based Body Composition Analysis}

Lee et al. [13] developed an FCN to segment skeletal muscle using $250 \mathrm{CT}$ scans from lung cancer patients. This network achieved high test accuracy (Dice: $0.93 ; n=$ 150 CT scans); however, the authors observed decreased performance in obese patients (Dice: 0.92 ) likely due to subcutaneous soft tissue edema being segmented as muscle [13]. Recently, Dabiri et al. [17] developed a combined FCN and UNet architecture for segmentation of skeletal muscle using three datasets (totaling 6,221 CT scans) from multiple different cancer types. This network demonstrated high accuracy (Dice: 0.98 ) on a large test set of 2,958 CT scans. Similar approaches have also been applied to segmenting adipose tissue [17]. Wang et al. [14] created a segmentation network using data from 40 (20 training and 20 test) ovarian cancer patients which demonstrated accurate segmentation of visceral (Dice: 0.92 ) and subcutaneous (Dice: 0.98 ) adipose tissue on test patients. Several recent publications have demonstrated that skeletal muscle, visceral adipose tissue, and subcutaneous adipose tissue can be accurately segmented within the same neural network $[15,16]$, which may provide improved computation speed for body composition analysis. Overall, these networks provide excellent segmentation accuracy (Table 1) while drastically improving the speed of analysis (e.g., 4,000 CT scans require approximately $15 \mathrm{~min}$ of analysis time), demonstrating that the laborious task of manual segmentation of CT scans for body composition analysis can be automated with high success.

While several publications have demonstrated good generalizability of their neural networks on test datasets for body composition analysis of CT scans (some using large datasets), neither the networks nor training data 
have been made publicly available, limiting the implementation and reproducibility of these approaches. Release of an anonymous dataset of segmented CT scans would enable direct comparisons and confirmation of the reproducibility of these networks.

\section{Moving Forward: What Is the Future of Deep Learning and Body Composition in Clinical Settings?}

These advances in the automated segmentation of CT scans for body composition analysis may aid clinicians and researchers in performing large-scale research studies investigating the influence of body composition in health and disease. While the current role of body composition in clinical settings is typically limited to retrospective research, if future studies are able to leverage these automated approaches for prospective body composition research, the prognostic role of body composition may be further validated as a clinically important metric to consider in the decision-making process (e.g., dose chemotherapy based on skeletal muscle mass to minimize the risk of chemotherapy toxicity). However, given the "black box" nature of neural networks, it will become critical to examine how health care providers interact with these systems. Visual confirmation of the segmentation results during routine $\mathrm{CT}$ interpretation by clinicians may be useful for both ensuring accuracy and instilling confidence in the results for treatment planning and clinical decisions. Ultimately, if body composition features emerge as an important metric in the prognosis or treatment for clinical populations, it will be necessary to evaluate whether these automated analysis systems are beneficial to clinicians and patients in improving outcomes.

\section{Disclosure Statement}

The authors have no conflicts of interest to declare.

\section{Funding Sources}

The author received no specific funding for this work.

\section{References}

1 Prado CM, Purcell SA, Alish C, Pereira SL, Deutz NE, Heyland DK, et al. Implications of low muscle mass across the continuum of care: a narrative review. Ann Med. 2018 Dec; 50(8):675-93

2 Vrieling A, Kampman E, Knijnenburg NC, Mulders PF, Sedelaar JPM, Baracos VE, et al. Body composition in relation to clinical outcomes in renal cell cancer: a systematic review and meta-analysis. Eur Urol Focus. 2018 Apr; 4(3):420-34.

3 Moisey LL, Mourtzakis M, Cotton BA, Premji T, Heyland DK, Wade CE, et al.; Nutrition and Rehabilitation Investigators Consortium (NUTRIC). Skeletal muscle predicts ventilator-free days, ICU-free days, and mortality in elderly ICU patients. Crit Care. 2013 Sep; 17(5):R206.

4 Paris M, Mourtzakis M. Assessment of skeletal muscle mass in critically ill patients: considerations for the utility of computed tomography imaging and ultrasonography. Curr Opin Clin Nutr Metab Care. 2016 Mar;19(2): 125-30.

5 Shen W, Punyanitya M, Wang Z, Gallagher D, St-Onge MP, Albu J, et al. Total body skeletal muscle and adipose tissue volumes: estimation from a single abdominal cross-sectional image. J Appl Physiol (1985). 2004 Dec;97(6): 2333-8

6 Sinclair A, Morrison A, Young C, Pyke L. The Canadian Medical Imaging Inventory, 2017. Ottawa: CADTH; 2018.
7 Popuri K, Cobzas D, Esfandiari N, Baracos V, Jägersand M. Body composition assessment in axial CT images using FEM-based automatic segmentation of skeletal muscle. IEEE Trans Med Imaging. 2016 Feb;35(2):512-20.

8 Patterson J, Gibson A. Deep Learning: A Practitioner's Approach. Sebastopol: O'Reilly Media; 2017.

9 LeCun Y, Bengio Y, Hinton G. Deep learning. Nature. 2015 May;521(7553):436-44.

10 LeCun Y, Bottou L, Bengio Y, Haffner P. Gradient-Based Learning Applied to Document Recognition. Proc IEEE. 1998;86(11):2278324.

11 Long J, Shelhamer E, Darrell T. Fully convolutional networks for semantic segmentation. Proc IEEE Comput Soc Conf Comput Vis Pattern Recognit. 2015:3431-40.

12 Ronneberger O, Fischer P, Brox T. U-Net: convolutional networks for biomedical image segmentation. In: Medical image computing and computer-assisted intervention - MICCAI 2015. Lecture Notes in Computer Science, vol 9351. Cham: Springer; 2015. p. 23441.

13 Lee H, Troschel FM, Tajmir S, Fuchs G, Mario J, Fintelmann FJ, et al. Pixel-Level Deep Segmentation: Artificial Intelligence Quantifies Muscle on Computed Tomography for Body Morphometric Analysis. J Digit Imaging. 2017 Aug;30(4):487-98.

14 Wang Y, Qiu Y, Thai T, Moore K, Liu H, Zheng B. A two-step convolutional neural network based computer-aided detection scheme for automatically segmenting adipose tissue volume depicting on CT images. Comput Methods Programs Biomed. 2017 Jun; 144:97-104.

15 Bridge CP, Rosenthal M, Wright B, Kotecha G, Fintelmann F, Troschel F, et al. Fully-automated analysis of body composition from CT in cancer patients using convolutional neural networks. In: Stoyanov D, et al., editors. OR 2.0 context-aware operating theaters, computer assisted robotic endoscopy, clinical image-based procedures, and skin image analysis. Lecture Notes in Computer Science, vol 11041. Cham: Springer; 2018. p. 204-13.

16 Weston AD, Korfiatis P, Kline TL, Philbrick KA, Kostandy P, Sakinis T, et al. Automated Abdominal Segmentation of CT Scans for Body Composition Analysis Using Deep Learning. Radiology. 2019 Mar;290(3):66979.

17 Dabiri S, Popuri K, Cespedes Feliciano EM, Caan BJ, Baracos VE, Beg MF. Muscle segmentation in axial computed tomography (CT) images at the lumbar (L3) and thoracic (T4) levels for body composition analysis. Comput Med Imaging Graph. 2019 Jul;75: $47-55$.

18 Burns JE, Yao J, Chalhoub D, Chen JJ, Summers RM. A machine learning algorithm to estimate sarcopenia on abdominal CT. Acad Radiol. 2019 May:S1076-6332(19)30165-5 [Epub ahead of print]. 